\title{
Quantum Contributions and Violations of the Classical Law of Mass Action
}

\author{
H. L. GRUBIN ${ }^{\mathrm{a}}$ and T. R. GOVINDAN ${ }^{\mathrm{b}}$ \\ ${ }^{a}$ Scientific Research Associates, Inc., Glastonbury, Connecticut \\ ${ }^{b}$ Applied Research Laboratory, State College, Pennsylvania
}

\begin{abstract}
The law of mass action refers to the product of concentration of reactants, which for a particular material is a function only of temperature. For non-degenerate semiconductors the law of mass action is represented by the product $N P=N_{C} N_{V} \exp -E_{G} / k_{B} T$. Through solutions to the quantum Liouville equation, it is determined that the law of mass action is generally invalid.
\end{abstract}

\section{INTRODUCTION}

Classically, for a specific material, non-degenerate statistics and equilibrium, the electron density, $N=N_{C}$ $\exp -\left[\left(E_{C}-E_{F}\right) / k_{B} T\right]$, and hole density, $P=N_{V}$ exp$\left[\left(E_{F}-E_{V}\right) / k_{B} T\right]$, yield, a temperature dependent classical law of mass action ${ }^{1,2}: N P=N_{C} N_{V} \exp -E_{G} / k_{B} T$.

Quantum mechanically, it is known, Wigner ${ }^{3}$, that modifications to the classical density-potential energy relation arise from gradients in charge density, which represent quantum mechanical attractive and repulsive forces. In particular, this relation may be expressed as ${ }^{4,5}$ :

(1) $\quad N=N_{C} \exp -\left[E_{C}(\mathbf{x})-E_{F}\right)$

$$
\left.+\left(\hbar^{2} / 6 \mathrm{~m}\right)(1 / \sqrt{ } N) \nabla^{2} \sqrt{ } N\right] / k_{B} T
$$

and strong departures from the classical mass action law occur when the density curvature (along with a similar expression for holes) is of the order of: $1\left(\nabla^{2}\right.$ $\sqrt{ } N) / \sqrt{ } N \approx \approx\left(6 \mathrm{~m} /{ }^{2}\right)\left(E_{C}(\underline{\mathrm{x}})-E_{F}\right)$.

\section{Modification of the Law of Mass Action- Approximate and Numerical Aspects}

Approximate analyses, via the quantum-corrected hydrodynamic equations (QHD) $)^{5,6,7,8}$, for non-uni- form electric fields, yields a quantum corrected law of mass action:

$$
\begin{aligned}
N P=N_{C} N_{V} \exp -\beta & {\left[E_{G}+\frac{\partial^{2} E_{C}}{\partial x^{2}}\left(\frac{\lambda_{e}^{2}-\lambda_{h}^{2}}{6}\right)\right.} \\
& \left.-\left(\frac{\beta}{2}\right)\left(\frac{\partial E_{C}}{\partial x}\right)^{2}\left(\lambda_{e}^{2}+\lambda_{h}^{2}\right)\right]
\end{aligned}
$$

which with some manipulation can be placed in the form of density curvatures, as in equation (1). Equation (2), consistent with equation (1), demonstrates that departures from the classical relation are determined primarily by the form, rather than the magnitude, of the potential energy. In equation (2), the thermal deBroglie wavelengths of electrons, $\lambda_{e}{ }^{2}=\hbar^{2} / 2 m_{e} k_{B} T$, and holes, $\lambda_{h}{ }^{2}=\hbar^{2} / 2 m_{h} k_{B} T$ have been introduced. Equation (2) indicates that for a PN junction, where the potential energy varies monotonically and where the sign of the curvature is different at the boundaries of the junction region, there will be differences in the shape of the spatial dependence of the $N P$ product.

Numerical results, showing the violation of the classical mass action law are obtained from the quan- 


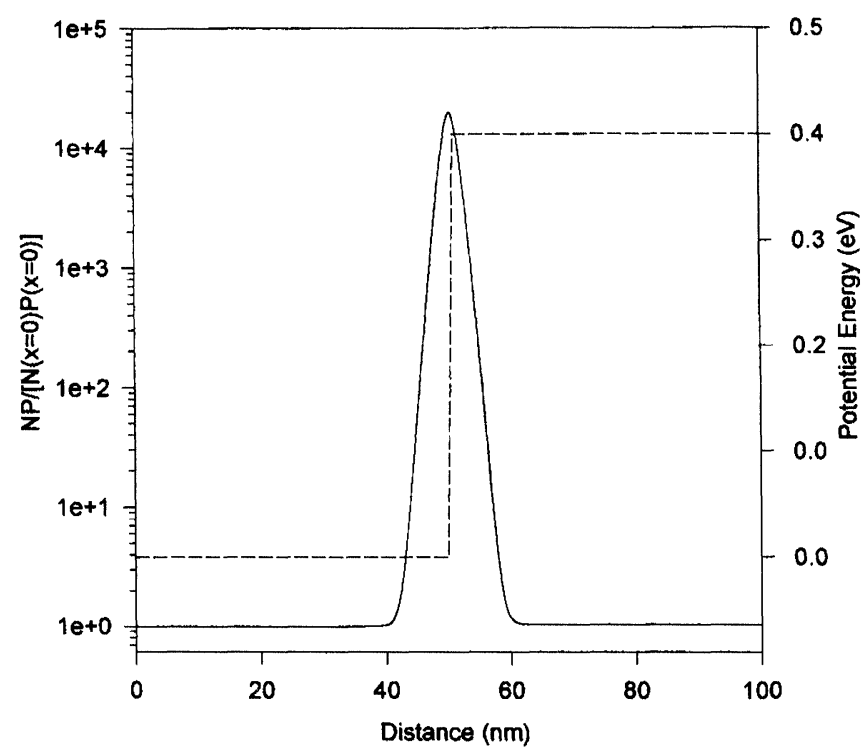

FIGURE 1 NP product and input conduction band energy. This calculation is performed for a background donor concentration of $10^{24} / \mathrm{m}^{3}$; the acceptor concentration was zero. The potential undergoes an abrupt change at the $50 \mathrm{~nm}$ point

tum Liouville equation, $i \hbar d \rho_{o p}(t) / d t=\left[H, \rho_{o p}(t)\right]$, in the coordinate representation ${ }^{9}$ :

$$
\begin{gathered}
\left(\frac{\hbar^{2}}{2 m_{e}}\right)\left(\frac{\partial^{2}}{\partial x^{2}}-\frac{\partial^{2}}{\partial x^{\prime 2}}\right) \rho_{e}- \\
\left(E_{C}(x)-E_{C}\left(x^{\prime}\right)\right) \rho_{e}=0 \text { (electrons) } \\
\left(\frac{\hbar^{2}}{2 m_{h}}\right)\left(\frac{\partial^{2}}{\partial x^{2}}-\frac{\partial^{2}}{\partial x^{\prime 2}}\right) \rho_{h} \\
\quad+\left(E_{V}(x)-E_{V}\left(x^{\prime}\right)\right) \rho_{h}=0 \text { (holes) }
\end{gathered}
$$

The solutions to equation (3) are obtained for Boltzmann-like boundary conditions:

$$
\begin{array}{ll}
\rho_{e}\left(x, x^{\prime}\right)=N_{C} \exp - & \\
{\left[\beta\left(E_{C}-E_{F}\right)+\left(x-x^{\prime}\right)^{2} / 4 \lambda_{e}^{2}\right]} & \text { (electrons) } \\
\rho_{h}\left(x, x^{\prime}\right)=N_{V} \exp - & \\
{\left[\beta\left(E_{F}-E_{V}\right)+\left(x-x^{\prime}\right)^{2} / 4 \lambda_{h}^{2}\right]} & \text { (holes) }
\end{array}
$$

which for uniform fields solutions are consistent with the classical law of mass action.

\section{Numerical Results: Non-Self Consistent Calculations}

We illustrate the departure from the classical law of mass action with non-self-consistent solutions to equation (3) for the situation where the conduction and valence bands are subject to identical changes in potential energy. The parameters chosen for the simulation were taken to maximize the accuracy of the numerical calculation. The simulation region was $100 \mathrm{~nm}$, and the density matrix was solved on a uniform $1000 \times 1000$ grid. The simulations were performed for a band-gap of leV, an electron effective mass of $0.067 \mathrm{~m}$, and holes with the same effective mass.

Figure 1 displays results for a $400 \mathrm{meV}$ step change in potential energy. According to equation (2) this should lead to an increase in the NP product over the classical result. This is illustrated in figure (1) through solutions to the quantum Liouville equation. Any departure from a constant value of $N P$ represents a violation of the classical law of mass action; and from figure 1, this departure is approximately four orders of magnitude. Since it may be argued that in a PN junction calculation the density gradients are such that it is unlikely that the potential energy will vary over an interval as small as that represented by figure 1, weaker slope calculations were also considered.

Increasing the region over which the potential energy undergoes a $400 \mathrm{meV}$ change results in a shallower slope. The deviation from the classical law of 


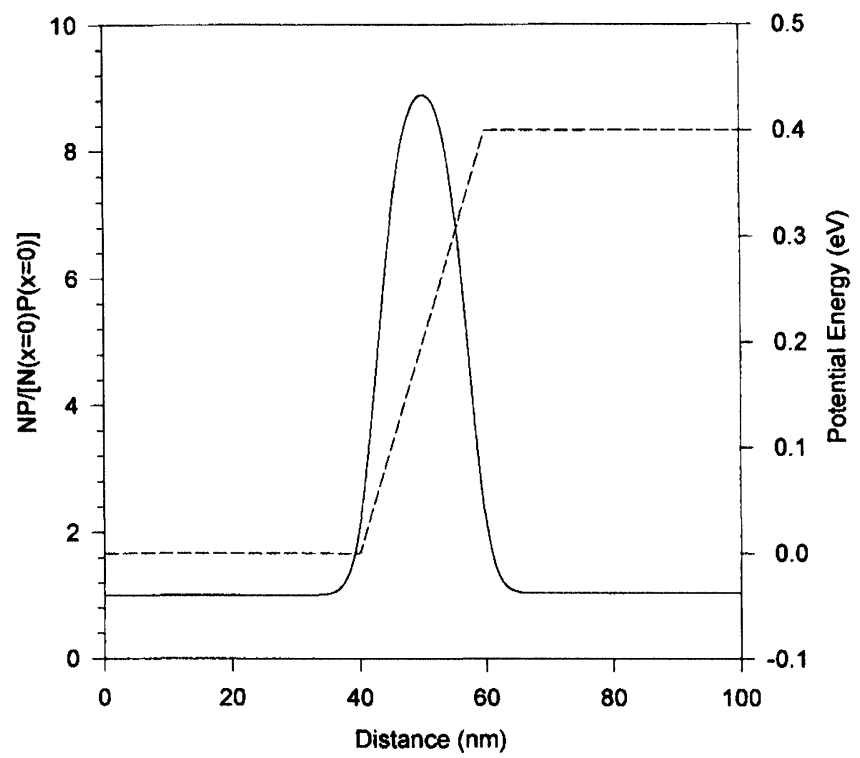

FIGURE 2 As in figure 1 but with a different potential energy. The NP product is on a linear scale. The peak value of the NP is significantly lower than that of figure 1 (The field is $200 \mathrm{keV} / \mathrm{cm}$.)

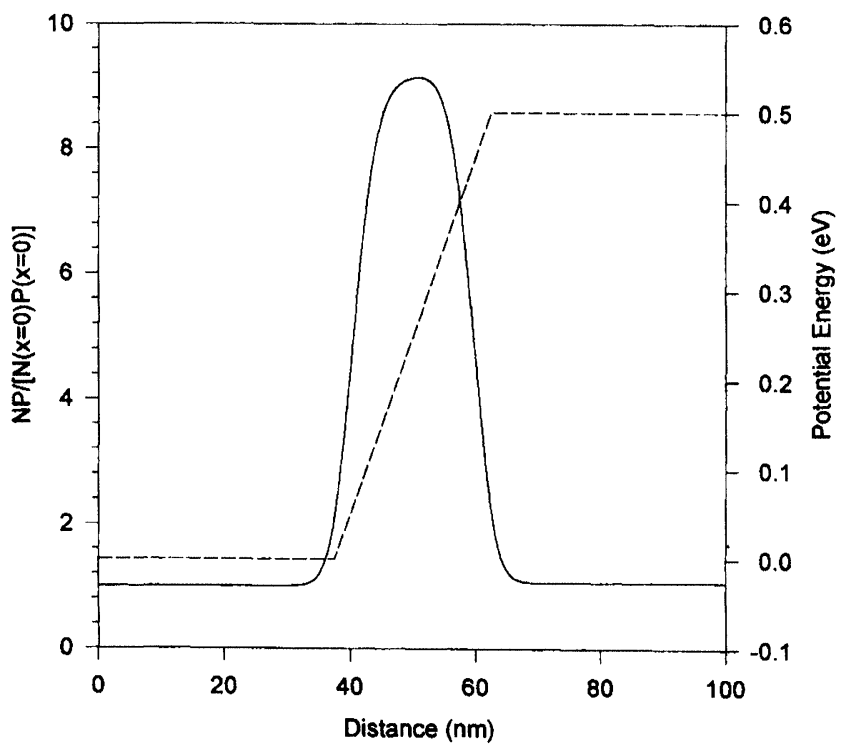

FIGURE 3 As in figure 2, with similar slope, but with the beginning and end points extended. The NP product, shown on a linear scale, has a peak value within the interior that is approximately the same as that offigure 2 , but extends over a larger region 
mass action as indicated by equation (2), is smaller. This is displayed in figure 2 where the potential energy changes value over a region of $20 \mathrm{~nm}$.

The change in magnitude of the potential energy is only one contribution to the alteration of the classical law of mass action, and perhaps a weak contribution. The quantum corrected mass action law represented approximately by equation (2) indicates that the shape of the spatial dependence of the potential energy is the determining factor in the departure of the law of mass action from equation (1). As seen in figure 3, keeping the slope of the potential energy fixed, but extending it over a larger region, such that the maximum value of the potential energy increases to $500 \mathrm{meV}$ has the primary effect of extending the region for the departure from the law of mass action. It appears that that magnitude of the departure is relatively unaltered.

\section{CONCLUSIONS}

Simple examples using the quantum Liouville equation in the coordinate representation, subject to the imposition of Boltzmann statistics, points to the fact that the law of mass action does not generally apply. The departure will be greatest in those materials having small effective mass. This result clearly has implications for the design of bipolar and heterostructure bipolar transistors in that the densities expected within specific regions are likely to depart from classical expectations when the critical region is small enough in scale. The consequences of this for design of devices remains to be examined. Self-consistent calculations have also been performed, and for parameters appropriate to GaAs, with results that are consistent with the above discussion ${ }^{10}$.

\section{Acknowledgements}

This work was supported by the Army Research Office and the Office of Naval Research.

\section{References}

[1] Kittel, C., and H. Kroemer, Thermal Physics, $2^{\text {nd }}$ Edition, W. H. Freeman and Co., San Francisco (1980)

[2] For degenerate material NPis a function of concentration as well as temperature.

[3] Wigner, E P., Phys. Rev. 40, 749, (1932)

[4] Ancona, M. A. and G. J. Iafrate, Phys. Rev. B39 9536 (1989)

[5] Grubin, H. L. and J. P. Kreskovsky, Sol. State Electron., 32, 1071 (1989)

[6] Grubin, H. L., T. R. Govindan, J. P. Kreskovsky and M. A. Stroscio, Sol. State Electron., 36, 1697 (1993)

[7] Zhou, J. R. and D. K. Ferry, IEEE Trans. Electron Dev. 39, 1793 (1992)

[8] Gardner, C. L. "The Quantum Hydrodynamic Model for Semiconductor Devices," SIAM J. Appl. Math 54 (1994) pp. 409-427.

[9] Grubin, H. L. Density Matrix Simulations of Semiconductor Devices, In Quantum Transport in Ultrasmall Devices D. K. Ferry, H. L. Grubin, C. Jacoboni an A.P. Jauho, Plenum Pres, NY (1995)

[10] Grubin, H. L. To be published. 

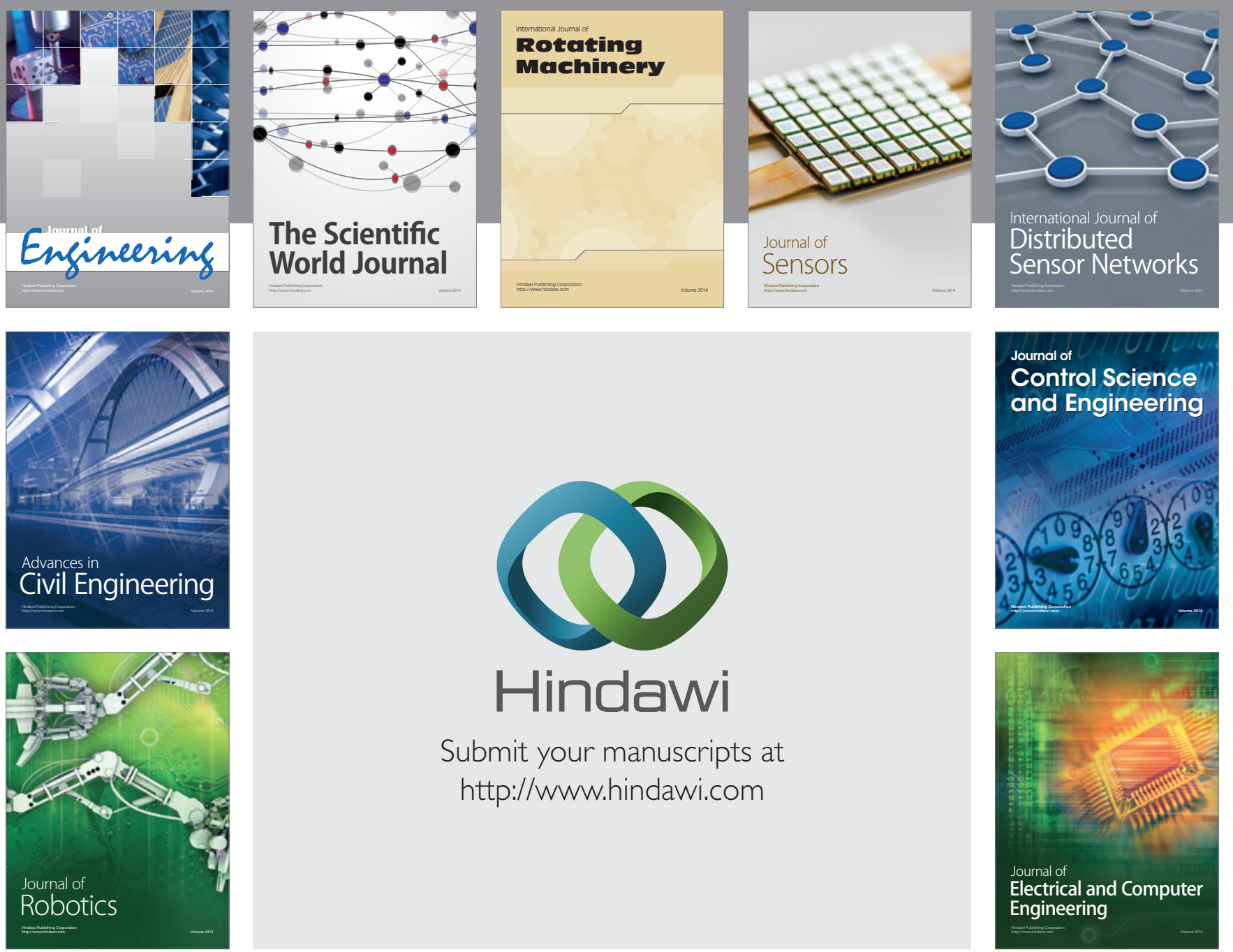

Submit your manuscripts at

http://www.hindawi.com
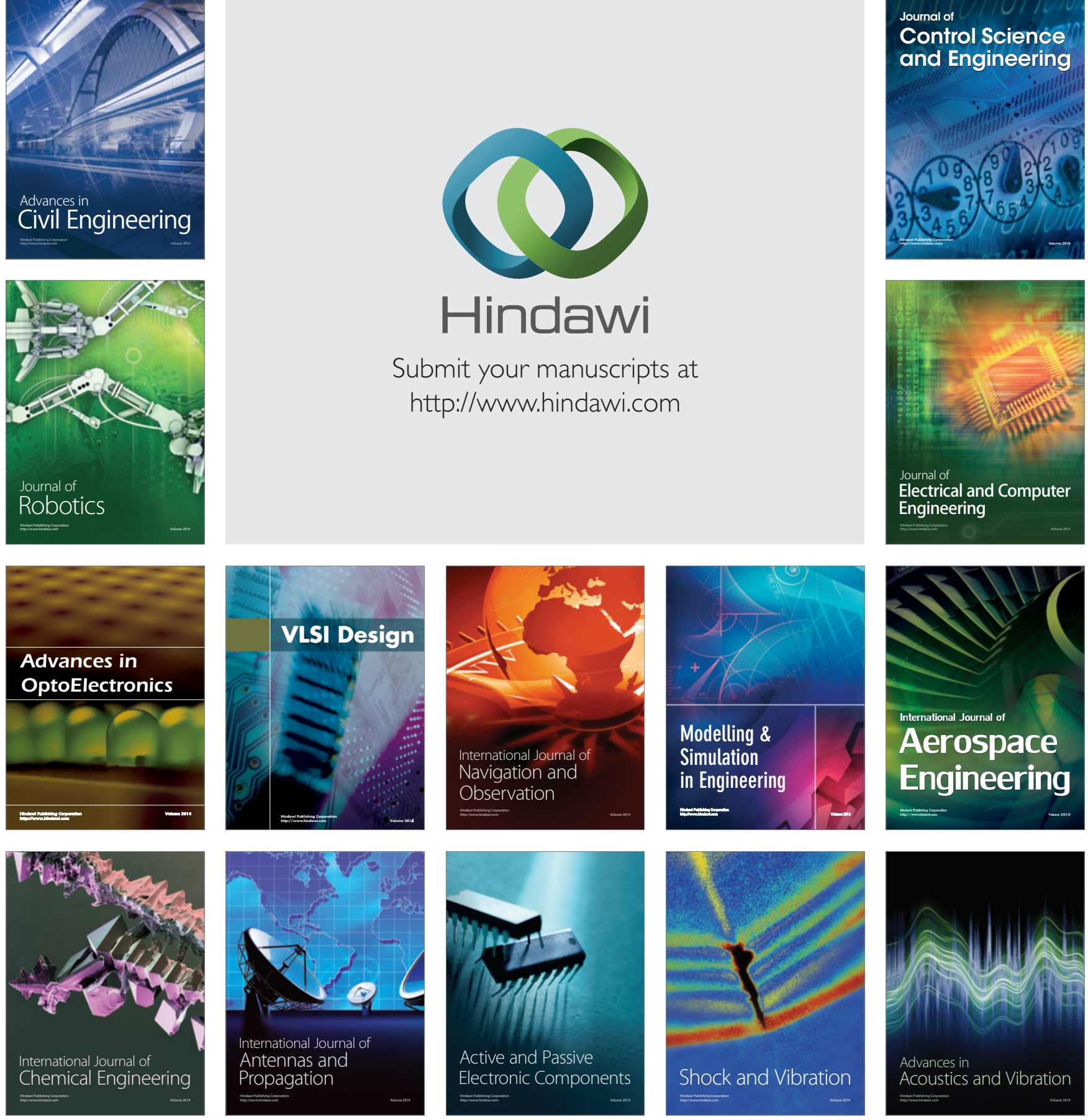